\title{
ARTIGOS EFICIÊNCIA DO TRATAMENTO ANAERÓBIO NA REMOÇÃO DE
HORMÔNIOS VETERINÁRIOS DO DEJETO LÍQUIDO SUÍNO
}

\author{
Gotardo R*, Pinheiro, $A^{* *}$, Kaufmann $\mathrm{V}^{* * *}$
}

Resumo

A contribuição de hormônios ao meio ambiente está ligada diretamente à eficiência da remoção de tais moléculas nas plantas de tratamento de águas residuárias. A remoção depende das características do efluente e dos processos adotados. O dejeto líquido suíno é o principal contribuinte de hormônios entre os resíduos do agronegócio. Sua concentração pode variar entre empresas integradoras, manejo, fase de criação, sexo e idade dos animais. Atualmente, a maior parte dos dejetos suínos é armazenada em esterqueiras por um período de 120 dias estabelecido pela legislação, e é posteriormente aplicada em lavouras como fertilizantes. Diante disso, neste trabalho objetivou-se avaliar a variabilidade das concentrações de hormônios durante a fase de terminação dos suínos, para diferentes manejos de empresas integradoras e a eficiência de remoção no sistema de tratamento (esterqueiras). O trabalho consistiu na seleção de três granjas produtoras de suínos de diferentes empresas, com criação de suínos na fase de terminação, instaladas no Município de Piratuba, SC. As coletas foram realizadas entre fevereiro e maio de 2013, durante o período de terminação dos suínos, que variou de 115 a 120 dias. As amostras foram coletadas em triplicata nas calhas coletoras de dejeto (resíduo líquido não tratado), e uma última coleta foi realizada na esterqueira após os 120 dias de armazenamento. Para cada uma das amostras foi avaliada a concentração dos hormônios 17a-estradiol, 17 $\beta$-estradiol, 17a-etinilestradiol, progesterona e mestranol. Nota-se que a tecnificação influenciou nas concentra-

\footnotetext{
* Mestre em Engenharia Ambiental pela Fundação Universidade Regional de Blumenau; doutorando em Engenharia Ambiental pela Universidade Regional de Blumenau; Rua Antônio da Veiga, 140, Itoupava Seca, 89012-900, Blumenau, Santa Catarina, Brasil; rafael. piratuba@yahoo.com.br

** Pós-doutor no Institut de Mécaniques de Fluides de Toulouse; Doutor em Física e Química Ambiental pelo Institut National Polytechnique de Toulouse; pinheiro@furb.br

*** Doutor em Recursos Hídricos e Saneamento Ambiental pelo Instituto de Pesquisas Hidráulicas na Universidade Federal do Rio Grande do Sul; Mestre em Engenharia Ambiental pela Universidade Regional de Blumenau; ambitec.amb@gmail.com.br
} 
ções médias dos hormônios analisados. A molécula 17a-estradiol apresentou um aumento em suas contrações durante o ciclo. As demais moléculas não tiveram variação significativa ou não foram quantificadas ao longo do tempo. As esterqueiras apresentaram eficiência de 8,93\% na remoção das concentrações de 17a-estradiol; em compensação, ocorreu um aumento de 54,21\% nas concentrações de $17 \beta$-estradiol. Acredita-se que durante o processo de tratamento pode ter ocorrido conversão do $17 \alpha$-estradiol em $17 \beta$-estradiol.

Palavras-chave: Suinocultura. Poluentes emergentes. Tratamento de águas residuárias.

\section{Efficiency of the anaerobic treatment in veterinary hormones removal of the liquid swine manure}

\section{Abstract}

The contribution of hormones to the environment is directly linked to the efficiency of removal of such molecules in wastewater treatment plants. The removal depends on the characteristics of the effluent and the processes adopted. The liquid swine manure is the main contributor of hormones between residues of agribusiness. Its concentration may vary among integrators, management, design phase, sex and age of the animals. Currently, most of the manure is stored in ponds for a period of 120 days, established by law, and it is subsequently applied to cropland as fertilizer. Thus, in the study it was aimed to assess variability in hormone levels during the swine finishing phase, for different management integration companies and the removal efficiency in the treatment system (ponds). The work consists of selecting three pig producer farms of different companies, with production of pigs in the finishing phase, installed in the city of Piratuba, SC. The samples were collected between February and May 2013, during the period of termination of the pigs, which ranged from 115 to 120 days. The samples were collected in triplicate in manure collecting gutters (liquid waste untreated), and a final gathering was accomplished in the dunghill after 120 days of storage. To each sample, it was evaluated the concentrations of hormones: $17 \alpha$-estradiol, 17 $\beta$-estradiol, $17 \alpha$-ethinylestradiol, mestranol and progestogen. It is observed that the technification influenced the average concentrations of the analyzed hormones. The $17 \alpha$-estradiol molecule showed an increase in its contractions during the cycle. The other molecules had no significant variation or were not quantified over time. The ponds had $8.93 \%$ efficiency in removing the concentrations of $17 \alpha$-estradiol; on the other hand, there was an increase of $54.21 \%$ in concentrations of $17 \beta$-estradiol. It is believed that during the treatment process it may have occurred conversion of $17 \alpha$-estradiol in $17 \beta$-estradiol.

Keywords: Swine. Emerging pollutants. Wastewater treatment.

\section{INTRODUÇÃO}

A suinocultura é uma atividade agropecuária de fundamental importância para o setor econômico brasileiro. Ela se desenvolveu rapidamente nos últimos 30 anos, por meio da modernização do setor, alcançando níveis elevados de produtividade. ${ }^{1}$ Atualmente, o Brasil ocupa a posição de maior 
exportador mundial de carne suína. No primeiro trimestre de 2015 foram abatidos 9,17 milhões de suínos, gerando um faturamento de 197,84 milhões de dólares em exportações. ${ }^{2}$

Embora o desenvolvimento da cadeia produtiva de suínos gere grandes benefícios à sociedade, ela também provoca sérios impactos ao meio ambiente. ${ }^{3}$ De acordo com Zhang, Zhu, King e $\mathrm{Li}^{4}{ }^{4} \mathrm{a}$ suinocultura combina alta densidade populacional em pequenas propriedades com o alto volume de dejetos produzidos por área. Em seu trabalho, Angonese, Campos, Palacio e Szymanski ${ }^{5}$ relatam que o alto volume de dejetos produzidos diariamente pelos animais em pequenas áreas é um dos maiores problemas do confinamento de suínos. Segundo Beli, Hussar e Hussar, ${ }^{6}$ um suíno produz, em média, cerca de 2,35 kg de efluente sólido, e após ser misturado com a parte líquida (urina e água), o volume aumenta para $5,80 \mathrm{~kg}$.

Com o objetivo de melhorar o desenvolvimento dos animais e, consequentemente, a produtividade, grande parte dos produtores adota programas nutricionais, que visam ao aumento da economia mediante o equilíbrio entre o fornecimento de ração e as exigências nutricionais dos animais. Por isso, diversas substâncias como nutrientes, hormônios e antibióticos têm sido encontradas nos dejetos animais. ${ }^{7}$ Por exemplo, Liu, Ying, Zhou, Zhang, Chen e Lai, ${ }^{8}$ estudando uma granja de suínos no Sul da China, determinaram concentrações de $0,78 \mu \mathrm{g} \mathrm{L^{-1 }}$ de estrona, $0,259 \mu \mathrm{g} \mathrm{L^{-1 }}$ de $17 \beta$-estradiol, $0,35 \mu \mathrm{g} \mathrm{L}{ }^{-1}$ de $17 \alpha$-etinilestradiol e 2,47 $\mu \mathrm{g} \mathrm{\textrm {L } ^ { - 1 }}$ de progesterona. Yost et al., ${ }^{9}$ estudando uma lagoa de dejeto suíno, quantificaram concentrações de progesterona que variaram de 0,11 a $0,51 \mu \mathrm{g} \mathrm{L}^{-1}$. Em estudo com antibiótico em dejeto suíno na Austrália, Martinez-Carballo, Barreiro-González, Scharf e Gans $^{10}$ encontraram concentração de $29,0 \mu \mathrm{g} \mathrm{L}^{-1}$ para oxitetraciclina e $46,0 \mu \mathrm{g} \mathrm{L}^{-1}$ para clortetraciclina. Xian-Gang, Qi-Xing e $\operatorname{Lin}^{11}$ determinaram concentrações de 58,0 $\mu \mathrm{g} \mathrm{L}^{-1}$ de clortetraciclina e 81,0 $\mu \mathrm{g}$ $\mathrm{L}^{-1}$ de tetraciclina em dejeto animal. A molécula sulfametazina foi determinada em dejeto de bovinos confinados por Aust et al., ${ }^{12}$ com concentração acima de 10,0 $\mu \mathrm{g} \mathrm{L} \mathrm{L}^{-1}$.

O uso de hormônios na alimentação animal é restrito no Brasil. Diversas leis e normativas regulamentam e proíbem o uso de substâncias que tenham efeitos hormonais para estimular o crescimento e a eficiência alimentar dos animais. No entanto, diversos estudos apontam a presença de hormônios naturais e sintéticos em matrizes ambientais, como em águas e solo. ${ }^{13,14,15}$ Compostos como estrona e $17 \beta$-estradiol já foram encontrados em água superficiais de países como Austrália, EUA e China. ${ }^{16,17,18}$ A presença de hormônios em ambientes aquáticos é altamente impactante, pois afeta negativamente os organismos presentes e, consequentemente, as funções do seu sistema endócrino. ${ }^{19}$

Os hormônios não são liberados somente por meio da aplicação, como promotores de crescimento, mas também, de forma espontânea pelo organismo, e essa liberação varia em razão do sexo, do estado fisiológico e do desenvolvimento dos animais. ${ }^{20}$ As fases de gestação e desenvolvimento dos animais são as principais responsáveis pelo aumento na liberação de hormônios naturais. De acordo com D’Ascenzo et al., ${ }^{21}$ em mulheres com idade reprodutiva, a liberação de estrona pode variar entre 5,0 e 31,0 $\mathrm{mg} \mathrm{dia}^{-1}$ e de estradiol, entre 3,0 e 19,0 $\mathrm{mg} \mathrm{dia}^{-1}$. Porém, os níveis de estrona e estradiol aumentam no período de gravidez, alcançando 600,0 a 940,0 $\mathrm{mg} \mathrm{dia}^{-1}$ e 170,0 a 330,0 $\mathrm{mg} \mathrm{dia}^{-1}$, respectivamente. Para os hormônios sintéticos, como o 17a-etinilestradiol, usado por mulheres como 
contraceptivo em doses diárias de $10,0 \mathrm{mg}$, cerca de $60 \%$ são eliminados na urina e fezes. ${ }^{22}$ De acordo com Lange, Hartel e Meyer, ${ }^{23}$ as fêmeas no ciclo de ovulação intensa liberam cerca de 0,014 mg dia ${ }^{-1}$ de hormônios pela urina.

Em animais, o comportamento dessas moléculas é semelhante ao de humanos, e suas concentrações variam em razão da espécie, sexo, idade, ciclo circadiano e estado reprodutivo. ${ }^{2}$ No entanto, o setor suinícola é um dos contribuintes mais efetivo de moléculas hormonais, até mesmo maior do que em relação aos resíduos humanos. ${ }^{24}$ Esse fato também pode ser atribuído aos resíduos de origem animal que receberam muito menos tratamento e atenção do que os resíduos humanos. ${ }^{25}$ Já o dejeto líquido suíno tem sido usado como biofertilizante primário em lavouras agrícolas, principalmente na produção de grãos e forragens. Em particular, o uso dos dejetos na agricultura é fonte potencial de poluição a ambientes aquáticos, em razão do baixo tratamento que recebem e das altas concentrações transportadas aos corpos hídricos. ${ }^{26,27}$ Vários estudos afirmam que os hormônios presentes em dejetos animais são transportados dos campos agrícolas aos ambientes aquáticos por meio escoamento superficial e da lixiviação. ${ }^{28,29,30,31}$

Os estudos realizados para investigar a remoção de compostos químicos, normalmente, concentram-se nas estações de tratamento que utilizam sistemas de lodos ativados. ${ }^{32,33} \mathrm{~A}$ inferência da remoção de fármacos, entre eles os hormônios, pelos sistemas de tratamentos convencionais é confirmada por grande parte dos autores que realizam estudos na área de tratamento de efluentes. ${ }^{34,35,36}$

Em todo o mundo, são usadas as mais diversas formas de armazenamento e tratamento de dejeto líquido suíno. Na Espanha, Alemanha e Portugal, os dejetos são armazenados nas chamadas lagoas alinhadas. No Brasil, existem diversos sistemas de tratamento de dejetos, entre eles se destacam os biodigestores, as lagoas de estabilização, a compostagem e as bioesterqueiras. A grande maioria das granjas criadoras de suínos fazem uso das esterqueiras como forma de tratamento primário dos dejetos. ${ }^{37}$

Cada um dos sistemas adotados para o tratamento do dejeto líquido de suíno possui particularidades que podem variar a sua eficiência na remoção das moléculas hormonais. No dejeto suíno bruto, Furuichi, Kannan, Suzuki, Tanaka, Giesy e Masunaga ${ }^{38}$ encontraram, em seus estudos, concentrações de $17 \alpha$-estradiol e $17 \beta$-estradiol que alcançaram 0,665 e 1,25 $\mu \mathrm{g} \mathrm{L}^{-1}$, respectivamente. Em lagoas de armazenamento, Sarmah, Meyer e Boxall ${ }^{19}$ determinaram concentrações de 0,011e 0,058 $\mu \mathrm{g}$ $\mathrm{L}^{-1}$ de $17 \alpha$-estradiol e $17 \beta$-estradiol, respectivamente. Raman et al. ${ }^{40}$ quantificaram concentrações de $3,90 \mu \mathrm{g} \mathrm{L} \mathrm{L}^{-1}$ para o $17 \alpha$-estradiol e de $2,50 \mu \mathrm{g} \mathrm{L}^{-1}$ para o $17 \beta$-estradiol em lagoas de tratamento de dejetos de fêmeas em gestação.

O sistema de tratamento conhecido por esterqueira consiste no armazenamento primário dos dejetos, em que a parte sólida contida no dejeto precipita e se acumula no fundo da lagoa, formando o lodo. Durante esse processo ocorre a digestão anaeróbia, reduzindo as cargas poluentes. Combalbert e Hernandez-Raquet ${ }^{20}$ relatam que a redução dos estrógenos depende do tempo de armazenamento do dejeto, das condições físico-químicas dos hormônios, do processo de tratamento e, fundamentalmente, da atividade biológica. 
O armazenamento em esterqueira e posterior aplicação no solo é a forma mais tradicional no Brasil, principalmente em Santa Catarina. ${ }^{41}$ Estas aplicações ocorrem ao longo do ano e devem obedecer às diretrizes das legislações vigentes. ${ }^{42}$ Essa atribuição é dada em virtude do potencial degradador e principalmente do seu tempo de meia-vida no ambiente, que pode variar de horas a dias. Ying, Toze, Hanna, Yu, Dillon e Kookan ${ }^{43}$ e Ying, Kookana, Kumar e Mortimer ${ }^{44}$ destacam que os hormônios são degradados mais rapidamente em condições aeróbias, tornando-se mais persistentes em condições anaeróbias.

No Brasil, os estudos sobre poluição do meio ambiente por hormônios ou estrógenos são restritos e limitados a uma gama pequena de moléculas. Poucos trabalhos até o presente momento têm procurado quantificar as concentrações de hormônios veterinários nos dejetos animais. Geralmente, os estudos se voltam a avaliar a remoção dos compostos em estações de tratamento de esgotos, em águas superficiais ${ }^{45}$ e de abastecimento público. ${ }^{46,47}$ Além disso, pouco se conhece sobre a composição hormonal dos dejetos líquidos de suínos, assim como seu comportamento durante o ciclo de produção final dos animais, a eficiência de remoção por parte do tratamento adotado e a variação entre as técnicas de produção adotada.

Dessa forma, neste trabalho objetivou-se avaliar a variabilidade das concentrações de hormônios durante o período da fase de terminação dos suínos para diferentes manejos de empresas integradoras e a eficiência de remoção pelo sistema de esterqueiras anaeróbias.

\section{MATERIAIS E MÉTODOS}

O estudo compreendeu coleta e análise de amostras de águas residuárias produzidas na suinocultura, em diferentes intervalos de tempo na fase de terminação dos animais. O estudo foi realizado em três granjas produtoras de suínos, localizadas no Município de Piratuba, SC, região do Meio-Oeste catarinense. As características das granjas são apresentadas na Tabela 1.

Tabela 1 - Descrição da localização e características das propriedades estudadas ${ }^{\dagger}$

\begin{tabular}{cccc}
\hline Granja & 1 & 2 & 3 \\
\hline Localidade & Linha Planalto & Linha Hachamam & Lageado Mariano \\
Latitude & $27^{\circ} 27^{\prime} 14^{\prime}$ & $27^{\circ} 24^{\prime} 30^{\prime}$ & $27^{\circ} 26^{\prime} 54^{\prime}$ \\
Longitude & $51^{\circ} 47^{\prime} 49^{\prime}$ & $51^{\circ} 44^{\prime} 29^{\prime}$ & $51^{\circ} 41^{\prime} 32^{\prime}$ \\
Sistema de criação & Terminação & Terminação & Terminação \\
Número de animais & 1.010 & 1.480 & 420 \\
Empresa integradora & $\mathrm{X}$ & $\mathrm{X}$ & $\mathrm{Y}$ \\
Formulação de rações & 9 & 9 & 5 \\
Tecnificação & alta & alta & média \\
Número de esterqueiras & 3 & 3 & 3 \\
Capacidade total de armazenamento & 1.000 & 1.500 & 500 \\
$\left(\mathrm{~m}^{3}\right)$ & $120^{*}$ & $120^{*}$ & $120^{*}$ \\
Tempo de detenção dos dejetos (dias) & &
\end{tabular}

${ }^{\dagger} 120$ dias é o tempo mínimo de armazenamento nas esterqueiras, estabelecido pela IN n. 11 da Fatma. 
Cada uma das granjas possui três esterqueiras de diferentes tamanhos, como apresentado na Tabela 1. O número de esterqueiras e a capacidade em cada uma das propriedades é uma relação entre o número de animais e o tempo de detenção dos dejetos. O processo de tratamento dos dejetos ocorre na forma de batelada, em que o volume total de dejetos é destinado primeiro a uma esterqueira, e, a partir desta, os dejetos são destinados para a segunda, e assim sucessivamente. Dessa forma atende-se ao tempo de detenção hidráulica estabelecido pela legislação.

A tecnificação das propriedades foi classificada de acordo com as práticas adotadas durante o processo de produção dos animais. Entre as práticas consideradas estão nutrição, sanidade, manejo, instalações e equipamentos. E, assim, as propriedades foram classificadas em média ou alta tecnificação.

As amostragens foram realizadas mensalmente de fevereiro a maio de 2013 durante a fase de terminação dos animais em intervalos de 30 dias. O procedimento de coleta foi realizado a partir do momento em que os animais foram alojados até a saída para o abate, período que variou de 115 a 120 dias. As coletas foram realizadas nas calhas coletoras que transportam os dejetos ao sistema de tratamento, considerando-se que nesse caso os dejetos não tinham sofrido nenhum processo de tratamento. E, com o objetivo de avaliar a eficiência do sistema de tratamento, a última coleta foi realizada na saída da esterqueira, 120 dias após o início do armazenamento dos dejetos. Este período corresponde ao período de tempo estabelecido pela legislação, também chamado de tempo de detenção hidráulica. As amostragens dos dejetos na esterqueira foram realizadas sempre no mesmo local, sendo homogeneizados com o auxílio de um rodo antes da coleta.

Considerando-se que o tempo de terminação dos animais variou de 115 a 120 dias, foi possível realizar cinco coletas de dejetos em cada uma das granjas. Em cada amostragem, foram coletadas três amostras em diferentes pontos da calha, fazendo-se, assim, uma triplicata das amostras. Estas foram acondicionadas em frascos de polietileno com capacidade de $50 \mathrm{~mL}$, mantidas a uma temperatura média de $4{ }^{\circ} \mathrm{C}$ em caixa de isopor com gelo e, em seguida, enviadas ao laboratório de cromatografia da Universidade Regional de Blumenau (Furb) para os procedimentos analíticos.

Para a extração, utilizou-se $5 \mathrm{~g}$ de resíduos dissolvidos em $10 \mathrm{~mL}$ de uma solução 75\% (v/v) de metanol em água. Essa suspensão foi transferida para tubos de extração e exposta por 30 minutos a um aparelho micro-ondas Anton Paar Multiwave 3000. Todas as amostras foram analisadas por Cromatografia Líquida de Alta Precisão (HPLC) Dionex Ultimate $300{ }^{\circledR}$, e nelas foram determinadas as concentrações dos homônimos 17a-estradiol, 17 $\beta$-estradiol, 17 $\alpha$-etinilestradiol, progesterona e mestranol.

A determinação foi realizada pela metodologia adaptada de Almeida e Nogueira, ${ }^{48}$ utilizando um HPLC com detecção de DAD em injeção direta. A fase móvel consistiu em uma solução aquosa de Acetonitrila 10\% (v/v) e Acetonitrila P.A., em um gradiente de 0 a 100\% de Acetonitrila P.A. em 60

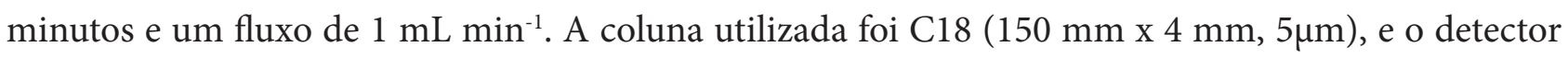
utilizou a faixa de onda de $200 \mathrm{~nm}$ para $17 \alpha$-estradiol, 17 $\beta$-estradiol, $17 \alpha$-etinilestradiol e mestranol, e $240 \mathrm{~nm}$ para progesterona. Em todas as análises, a temperatura da coluna foi mantida constante a 25 ${ }^{\circ} \mathrm{C}$, e o volume de injeção foi de $200 \mu \mathrm{l} \mathrm{min}^{-1}$. 
A avaliação dos resultados foi dividida em quantificado, detectado e não detectado (Tabela 2). Considerou-se que a molécula foi quantificada quando sua concentração foi superior ao limite de quantificação definido a partir das curvas de calibração. O valor detectado indicou que a molécula foi detectada, mas a concentração não foi suficiente para o equipamento determinar sua concentração na amostra. $\mathrm{O}$ analisado e não detectado indicou que ocorreu um sinal cromatográfico, mas não foi possível ser identificada a molécula.

Tabela 2 - Limites de quantificação e detecção $\left(\right.$ ng L $\left.^{-1}\right)$

\begin{tabular}{ccc}
\hline Moléculas & Limite de deteç̧ão & Limite de quantificação \\
\hline $17 \alpha$-estradiol & 1,28 & 3,89 \\
$17 \beta$-estradiol & 2,18 & 6,62 \\
$17 \alpha$-etinilestradiol & 0,86 & 2,60 \\
Mestranol & 1,70 & 5,16 \\
Progesterona & 1,37 & 4,16 \\
\hline
\end{tabular}

O limite de quantificação foi determinado pelo desvio padrão da curva de calibração multiplicado por 10, e o limite de detecção foi calculado pela declividade da curva de calibração multiplicado por 3,14, de acordo com o recomendado por Ribani, Bottoli, Collins, Jardim e Melo. ${ }^{49}$

Após análise das concentrações de entrada e saída do sistema de tratamento, os resultados foram comparados para se avaliar a eficiência de remoção dos hormônios pela esterqueira. A eficiência na remoção foi expressa de acordo com a seguinte equação:

$$
E f=\frac{C e-C s}{C e} \times 100
$$

em que,

$E f=$ eficiência de remoção do hormônio considerado (\%);

$\mathrm{Ce}=$ concentração média na entrada do sistema de tratamento $\left(\right.$ ng $\left.\mathrm{L}^{-1}\right)$;

$C s=$ concentração média na saída do sistema de tratamento $\left(n g \mathrm{~L}^{-1}\right)$.

Os resultados foram analisados e comparados estatisticamente pelo teste de Tukey a 5\% de significância com o software Statistica (Statsoft version 5.0).

\section{RESULTADOS E DISCUSSÃO}

As concentrações médias, máximas e mínimas dos hormônios determinados na entrada dos dejetos (calha coletora) no sistema de tratamento para as três granjas estão apresentadas na Tabela 3. As concentrações das moléculas hormonais analisadas variaram suas concentrações médias, máximas e mínimas entre as granjas. 
Tabela 3 - Concentrações médias, máximas e mínimas em ng $\mathrm{L}^{-1}$ determinadas na entrada do sistema de tratamento

\begin{tabular}{ccccccc}
\hline Granja & Atributo & $17 \alpha$-estradiol & $17 \beta$-estradiol & $17 \alpha$-etinilestradiol & Mestranol & Progesterona \\
\hline \multirow{2}{*}{1} & Média & $1.448,30$ & $1.499,87$ & 23,24 & 0,01 & 1,02 \\
& Máximo & $4.350,29$ & $3.424,51$ & 93,99 & 0,16 & 3,53 \\
& Mínimo & 168,55 & 686,13 & 0,00 & 0,00 & 0,00 \\
& Média & $1.748,53$ & $1.612,48$ & 0,64 & 0,00 & 1,05 \\
& Máximo & $4.235,87$ & $3.561,03$ & 4,11 & 0,00 & 2,20 \\
& Mínimo & 104,83 & 187,22 & 0,00 & 0,00 & 0,00 \\
& Média & $3.069,98$ & 159,56 & 67,74 & 0,01 & 0,55 \\
& Máximo & $6.442,24$ & 894,91 & 409,10 & 0,12 & 1,56 \\
& Mínimo & 549,91 & 0,00 & 0,00 & 0,00 & 0,15 \\
\hline
\end{tabular}

Os resultados das concentrações médias de 17a-estradiol para as Granjas 1 e 2 foram muito próximos, sendo 1.448,30 ng L-1 e 1.748,53 ng L-1 , respectivamente. A concentração média mais alta ocorreu na Granja 3, com valor de 3.069,98 ng L-1. Para o 17ß-estradiol, as maiores concentrações médias ocorreram para as Granjas 1 e 2, ficando com 1.499,87 ng L $\mathrm{L}^{-1}$ e 1.612,48 ng L $\mathrm{L}^{-1}$, respectivamente. Na Granja 3, a concentração máxima alcançou o valor de apenas 159,56 ng L-1. Os valores das concentrações determinadas neste trabalho estão muito acima dos 6,0 ng L $\mathrm{L}^{-1}$ estabelecidos por Bastos, Bevilacqua e Mierzwa, ${ }^{50}$ como a concentração máxima que não causa efeito adverso à saúde humana.

As altas concentrações de $17 \alpha$-estradiol e $17 \beta$-estradiol estão ligadas ao manejo dos animais, como a nutrição e os tratamentos veterinários. Diante disso, vale lembrar que as Granjas 1 e 2 possuem alta tecnificação, ou seja, fazem uso de um maior número de técnicas durante o processo de produção dos animais. Dessa forma, o maior número de formulações de rações e o número de procedimentos veterinários exigidos pelos programas sanitários, como o uso da imunocastração, podem ter alterado tais concentrações. A imunocastração é um procedimento veterinário realizado mediante a aplicação de uma vacina que induz a formação de anticorpos contra o $\mathrm{GnRH}$, os quais, ao se ligarem, impedem a secreção do hormônio folículo estimulante (FSH) e do hormônio luteinizante (LH), reduzindo a secreção de esteroides testiculares. Entre as vantagens dessa técnica estão a redução do odor sexual na carcaça, o aumento nas taxas de crescimento e a eficiência alimentar dos animais. Nesse caso, a tecnificação pode ter alterado significativamente as concentrações do $17 \beta$-estradiol. Em seus estudos, Reis Filho, Araújo e Vieira ${ }^{51}$ já consideravam o $17 \beta$-estradiol e o hormônio sintético $17 \alpha$-etinilestradiol como os de maior preocupação em relação ao meio ambiente, dada sua quantidade introduzida no meio. Essas substâncias são consideradas responsáveis por desencadear grande parte dos efeitos dos disruptores endócrinos.

Por meio das concentrações médias pode ser observado que o 17a-estradiol e o $17 \beta$-estradiol estão presentes no dejeto líquido suíno em altas concentrações, e, no caso deste estudo, o manejo dos animais adotado pelos produtores pode ter interferido significativamente nas médias, ou seja, as mudanças das diferentes formulações de rações e os procedimentos veterinários adotados podem ter alterado as concentrações médias para essas substâncias. 
As concentrações de 17a-estradiol foram sempre superiores às concentrações de $17 \beta$-estradiol. Esta tendência também foi determinada por Hutchins, White, Hudson e Fine ${ }^{52}$ em lagoas primárias de dejeto de porcas, onde a concentração de $17 \alpha$-estradiol foi de 1.300,00 ng L ${ }^{-1}$, e a de $17 \beta$-estradiol, um pouco menor apenas 200,00 $\mathrm{ng} \mathrm{L}^{-1}$. Em um estudo realizado por Raman et al., ${ }^{40}$ foram encontradas em lagoas de porcas em estágio de parição concentrações de 3.900,00 e 2.500,00 ng L-1 para 17a-estradiol e $17 \beta$-estradiol, respectivamente.

O 17a-etinilestradiol apresentou a maior variação nas concentrações médias entre as granjas analisadas. A concentração média mais alta foi de 409,10 ng L $\mathrm{L}^{-1}$ na Granja 3, e a menor foi de 4,11 ng $\mathrm{L}^{-1}$ na Granja 2. Estes resultados estão dentro das concentrações encontradas por Liu, Ying, Zhou, Zhang, Chen e Lai, ${ }^{8}$ os quais determinaram concentrações de 0 a 357,00 ng L ${ }^{-1}$ na parte líquida do dejeto suíno. A variação entre as concentrações médias para esta molécula demonstrou que a tecnificação não influenciou em suas concentrações. Então, considera-se que estas concentrações podem ser provenientes de adição externa, como o uso de medicamento veterinário específico. $\mathrm{O}$ mestranol e a progesterona não apresentaram concentração em níveis quantificáveis.

Na Tabela 4 são apresentadas as concentrações médias das moléculas analisadas na calha (entrada) aos 120 dias dos animais alojados (dejeto não tratado) e na esterqueira (saída) aos 120 dias do dejeto armazenado (dejeto tratado), mostrando a eficiência da remoção dos hormônios pela esterqueira.

A esterqueira apresentou diferença estatística significativa quanto a remoções para as moléculas $17 \alpha$-estradiol e $17 \beta$-estradiol. Os resultados mostraram que ocorreu remoção de $8,93 \%$ para $17 \alpha$-estradiol, mas para o $17 \beta$-estradiol, a esterqueira apresentou eficiência negativa na remoção, considerando-se que suas concentrações passaram de 71,22 para 109,83 ng L-1, o que representa um aumento de 54,21\%. Esse resultado concorda em partes com os estudos de Furuichi, Kannan, Suzuki, Tanaka, Giesy e Masunaga, ${ }^{38}$ no qual, em uma unidade de tratamento anaeróbio de dejeto suíno, detectou uma eficiência de remoção de $6 \%$ para $17 \alpha$-estradiol e de $65 \%$ para o $17 \beta$-estradiol. Ferreira ${ }^{53}$ relata em um de seus trabalhos a eficiência das plantas de tratamento na eliminação de grande parte do $17 \alpha$-estradiol durante o processo de tratamento. Baronti, Curini, Ascenzo, Corcia, Gintili e Chimica $^{54}$ também relataram a eficiência das plantas de tratamento na remoção de $85 \%$ para o estradiol.

Tabela 4 - Eficiência da esterqueira na remoção para as concentrações médias

\begin{tabular}{cccc}
\hline Hormônio $\left(\mathrm{ng} \mathrm{L}^{-1}\right)$ & Calha (120 dias) & Esterqueira (120 dias) & Eficiência na remoção (\%) \\
\hline $17 \alpha$-Estradiol & $4.362,81$ & $3.973,38$ & 8,93 \\
$17 \beta$-Estradiol & 71,22 & 109,83 & $-54,21$ \\
$17 \alpha$-Etinilestradiol & nd & nd & - \\
Mestranol & nd & nd & - \\
Progesterona & nd & nd & - \\
\hline
\end{tabular}

A remoção do $17 \alpha$-estradiol pode estar ligada ao tempo de detenção dos dejetos na esterqueira. O tempo de 120 dias pode ter sido insuficiente para a remoção total dessa molécula. Diversos estudos têm discutido o aumento da eficiência na remoção de hormônios com o tempo de detenção 
dos dejetos. ${ }^{55,56,57}$ Mas segundo Combalbert e Hernandez-Raquet, ${ }^{20}$ além do tempo de retenção hidráulica, as remoções de hormônios dependem da carga de sólidos, da temperatura, do potencial redox, do tamanho de partículas, da diluição pelas chuvas e, principalmente, do crescimento microbiano.

Cabe ressaltar que a eficiência de remoção pela esterqueira pode ser discutida e, até mesmo, questionada, quando se considera a conversão das moléculas em uma segunda molécula. Neste caso, a molécula pode provir da transformação ou degradação bioquímica de uma segunda molécula pertencente ao mesmo grupo químico. Assim, tem-se a hipótese de ter ocorrido a conversão do 17a-estradiol em $17 \beta$-estradiol ou em uma terceira molécula. Em estudos laboratoriais com resíduos da atividade leiteira, Raman et al. ${ }^{40}$ perceberam nos resultados uma rápida transformação de estradiol em estrona.

As moléculas mestranol e progesterona não resultaram em concentrações quantificáveis durante os 120 dias do alojamento dos animais, o que confirma as análises realizadas nas esterqueiras, onde as concentrações não foram quantificáveis. Esse resultado é muito importante, pois o mestranol, além de outros hormônios naturais e sintéticos, é causador de atividades estrogênicas em corpos hídricos. ${ }^{58}$ As baixas concentrações da progesterona está relacionada à ausência de fêmeas em períodos de gestação. Segundo Smith, ${ }^{59}$ o pico de produção da progesterona ocorre 12 dias após o início da gestação, mantendo-se constante até os 104 dias. ${ }^{60}$ Próximo ao parto, os valores são reduzidos significativamente, ${ }^{61}$ chegando a apenas $0,5 \mathrm{ng} \mathrm{mL} \mathrm{mL}^{-1}$ um dia após o parto, ${ }^{59}$ ou seja, os valores em períodos fora da gestação ficam abaixo dos níveis de quantificação. A afirmação desses autores vem corroborar esse resultado se se considerar que as granjas estudadas trabalham com suínos em terminação e não em sistemas de cria e recria.

Na Tabela 5 são apresentados e o comportamento e a evolução das concentrações médias de cada uma das moléculas analisadas durante a fase de terminação dos suínos. Nota-se que o 17a-estradiol aumentou sua concentração durante o período de alojamento. A concentração média passou de 374,27 ng L-1 , aos 30 dias, para 4.362,81 $\mathrm{ng} \mathrm{L}^{-1}$, ao completar os 120 dias do ciclo de alojamento.

O $17 \beta$-estradiol apresentou correlação negativa em relação ao $17 \alpha$-estradiol, visto que a concentração média de $17 \beta$-estradiol passou de 2.472,48 $\mathrm{ng} \mathrm{L}^{-1}$, aos 30 dias, para 71,22 ng L $\mathrm{L}^{-1}$, aos 120 dias. Este resultado está atribuído a dois aspectos: o tempo de meia-vida da molécula, considerado de dois a nove dias nos estudos de Reis e Filhos, ${ }^{51}$ e ao fato de suas concentrações não terem atingido o limite de quantificação do equipamento, que neste caso é de 6,62 $\mathrm{ng} \mathrm{L}^{-1}$. Alguns estudos abordam resultados muitos semelhantes, como China, Jiang et al., ${ }^{18}$ que, em estudos de canais fluviais na China, concluíram que as concentrações de $17 \beta$ - estradiol ficaram abaixo do limite de quantificação, e Lu, Song, Wang e Yan ${ }^{62}$ e Zuo, Zhang e Deng, ${ }^{63}$ que encontraram em seus estudos concentrações médias de 0,97 e $0,83 \mathrm{ng} \mathrm{L}^{-1}$, respectivamente. 
Tabela 5 - Evolução das concentrações médias $\left(n g \mathrm{~L}^{-1}\right)$ durante o período de terminação

\begin{tabular}{ccccc}
\hline \multirow{2}{*}{ Molécula } & \multicolumn{4}{c}{ Tempo do período de terminação $(\mathrm{d})$} \\
\cline { 2 - 5 } & 30 & 60 & 90 & 120 \\
\hline 17 a-estradiol & $374,27 \pm 308,19$ & $1.468,34 \pm 813,38$ & $2.719,42 \pm 1.506,60$ & $4.362,81 \pm 386,82$ \\
$17 \beta$-estradiol & $2.472,48 \pm 1.474,14$ & $653,78 \pm 306,02$ & $817,98 \pm 890,92$ & $71,22 \pm 107,50$ \\
17 -etinilestradiol & $97,20 \pm 166,12$ & $12,33 \pm 18,80$ & $11,77 \pm 26,26$ & nd \\
Mestranol & nd & nd & nd & nd \\
Progesterona & nd & & nd & nd \\
\hline
\end{tabular}

O 17a-etinilestradiol também apresentou redução da concentração média ao longo do ciclo, sendo zerada aos 120 dias. Este resultado também foi alcançado por Gadd, Tremblay e Northcott, ${ }^{64}$ que, em um estudo realizado com efluentes de uma lagoa de tratamento de dejetos animais, atribuíram a variação das concentrações de estrógenos livres às diferentes épocas e manejos.

Assim como nos demais parâmetros analisados, o mestranol e a progesterona não foram quantificados em nenhum momento do período de avaliação. A concentração de tais moléculas também pode ter ficada abaixo dos limites de quantificação do equipamento de análise, que atinge, no máximo, $5,16 \mathrm{ng} \mathrm{L}^{-1}$.

\section{CONCLUSÃO}

Os resultados mostram que o $17 \alpha$-estradiol e o $17 \beta$-estradiol são os principais poluentes entre os estrógenos avaliados, em razão da sua frequência nas amostras e das altas concentrações encontradas ao longo do ciclo dos animais.

A tecnificação adotada nas granjas influenciou a concentração do 17a-estradiol e do $17 \beta$-estradiol, considerando-se que as maiores concentrações estiveram presentes nas granjas com maiores exigências no manejo e, consequentemente, o uso constante de medicamentos veterinários.

A esterqueira mostrou-se com uma baixa eficiência na remoção do $17 \beta$-estradiol e ineficiente na remoção do 17 a-estradiol; porém, essa baixa eficiência ou ineficiência não podem ser completamente comprovadas, pois não foi avaliada a possível conversão bioquímica entre moléculas.

Durante o ciclo produtivo ou de alojamento dos suínos, as moléculas hormonais apresentam evoluções desiguais. O 17a-estradiol aumentou sua concentração durante o período de alojamento dos animais, enquanto as demais moléculas reduziram ou não foram quantificadas nas amostras coletadas.

\section{REFERÊNCIAS}

1. Instituto Brasileiro de Geografia e Estatística [Internet]. Indicadores IBGE: estatística da produção pecuária. jun. 2015 [acesso em 2016 fev 14]. Disponível em: http://www.ibge.gov.br/home/estatistica/ indicadores/agropecuaria/producaoagropecuaria/abate-leite-couro-ovos 201501 publ completa.pdf. 
2. Associação Brasileira de Indústria Produtora e Exportadora de Carne de Suínos [Internet]. 2012 [acesso em 2014 jan 03]. Disponível em: http://www.abipecs.org.br/uploads/relatorios/mercadoexterno/exportacoes/anuais/jan-nov-2013 jan-nov-2012.pdf.

3. Rocha, D. Tratamento dos dejetos da suinocultura [Internet]. [acesso em 2015 jan 27]. Disponível em: http://www.ambienteemfoco.com.br/?p=180/Delcio\%20Rocha.

4. Zhang Z, Zhu J, King J, Li W. A two-step fed SBR for treating swine manure. Process Biochem. 2006; 41(4):892-900.

5. Angonese AR, Campos AT, Palacio SM, Szymanski N. Avaliação da eficiência de um biodigestor tubular na redução da carga orgânica e produção de biogás a partir de dejetos de suínos. In: Anais do $6^{\circ}$ Congresso Internacional sobre Geração distribuída e Energia no Meio Rural, 2006; Campinas.

6. Beli E, Hussar GJ, Hussar DH. Redução de DQO e turbidez de efluente de uma unidade suinícola empregando Reator Anaeróbio Compartimentado (RAC) seguido de filtro biológico e filtro de areia. Eng. Ambiental. 2010; 7(1):5-19.

7. Adams CD. Treatment of antibiotics in swine wastewater. In: Aga DS. Fate of pharmaceuticals in the environment and in water treatment systems. New York: CRC Press; 2008.

8. Liu S, Ying G-G, Zhou L-J, Zhang RQ, Chen ZF, Lai HJ. Steroids in a typical swine farm and their release into the environment. Water Res. 2012; 46:3754-68.

9. Yost EE, Meyer MT, Dietze JE, Meissner BM, Worley-Davis L, Williams CM, et al. Comprehensive assessment of hormones, phytoestrogens, and estrogenic activity in an anaerobic swine waste lagoon. Environ. Sci. Technol. 2013; 47:13781-9.

10. Martínez-Carballo E, Barreiro-González C, Scharf S, Gans O. Environmental monitoring study of selected veterinary antibiotics in animal manure and soils in Austria. Environ. Pollut. 2007; 148(2):570-9.

11. Xiang-Gang H, Qi-Xing Z, Lin X. Determination of thirteen antibiotics residues in manure by solid phase extraction and high performance liquid chromatography. Chinese J. Anal. Chem. 2008; 36(9):1162-6.

12. Aust MO, Godlinski F, Travis GR, Hao X, Mcallister TA, Leinweber P et al. Distribution of sulfamethazine, chlortetracycline and tylosin in manure and soil of Canadian feedlots after sub therapeutic use in cattle. Environ. Pollut. 2008; 156:1243-51.

13. Bradley PM, Barber LB, Chapelle FH, Gray JL, Kolpin DW, Mcmahon PB. Biodegradation of 17a-estradiol, estrone and testosterone in stream sediments. Environ. Sci. Technol. 2010; 6(43):190210.

14. Casey FXM, Hakk H, Simunek J, Larsen GL. Fate and transport of testosterone in agricultural soils. Environ. Sci. Technol. 2004; 38(3):790-8. 
15. Chang H, Wan Y, Hu JY. Determination and source apportionment of five classes of steroid hormones in Urban Rivers. Environ. Sci. Technol. 2009; 20(43): 7691-8.

16. Hohenblum P, Gans O, Moche W, Scharf S, Lorbeer G. Monitoring of selected estrogenic hormones and industrial chemicals in groundwater's and surface waters in Austria. Sci. Total Environ. 2004; 333(1-3):185-93.

17. Benotti MJ, Trenholm RA, Vanderford BJ, Holady JC, Stanford BD, Snyder SA. Pharmaceuticals and endocrine disrupting compounds in U.S. drinking water. Environ. Sci. Technol. 2009; 43(3):597603.

18. Jiang WW, Yan Y, Ma M, Wang DH, Luo Q, Wang ZJ, et al. Assessment of source water contamination by estrogenic disrupting compounds in China. J. Environ. Sci. 2012; 24.

19. Kuster M, Díaz-Cruz S, Rossel M, Alda ML, Barceló B. Fate of selected pesticides, estrogens, progestogens and volatile organic compounds during artificial aquifer recharge using surface waters. Chemosphere. 2010; 79:880-6.

20. Combalbert S, Hernandez-Raquet G. Occurrence, fate, and biodegradation of estrogens in sewage and manure. Appl. Microbiol. Biot. 2010; 86(6):1671-92.

21. D’ascenzo G, Di Corcia A, Gentili A, Mancini R, Mastropasqua R, Nazzari M, et al. Fate of natural estrogen conjugates in municipal sewage transport and treatment facilities. Sci. Total Environ. 2003; 302:199-209.

22. Johnson AC, Williams RJA. Model to estimate influent and effluent concentrations of estradiol, estrone, and ethinylestradiol at sewage treatment works. Environ. Sci. Technol. 2004; 13(38):3649-58.

23. Lange IG, Hartel A, Meyer HHD. Evolution of estrogens functions in vertebrates. J. Steroid. Biochem. Mol. Biol. 2003; 83(1-5):219-26.

24. Johnson AC, Williams RJ, Matthiessen P. The potential steroid hormone contribution of farm animals to freshwaters, the United Kingdom as a case study. Sci. Total Environ. 2006; 362(1-3):16678.

25. Liu S, Ying G-G, Zhang R-Q, Zhou L-J, Lai H-J, Chen Z-F. Fate and occurrence of steroids in swine and dairy cattle farms with different farming scales and wastes disposal systems. Environ. Pollut. 2012; 170:190-201.

26. Khanal SK, Xie B, Thompson ML, Sung SW, Ong SK, Van Leeuwen J. Fate, transport, and biodegradation of natural estrogens in the environment and engineered systems. Environ. Sci. Technol. 2006; 21(40):6537-46.

27. Lee LS, Carmosini N, Sassman SA, Dion HM, Sepulveda MS. Agricultural contributions of antimicrobials and hormones on soil and water quality. Adv. Agron. 2007; 93:1-68. 
28. Finlay-Moore O, Hartel PG, Cabrera ML. 17 $\beta$-estradiol and testosterone in soil and runoff from grasslands amended with broiler litter. J. Environ. Qual. 2000; 29(5):1604-11.

29. Hanselman TAG, Raetz DA, Wilkie AC. Manure-borne estrogens as potential environmental contaminants: A review. Environmental Science \& Technology. 2003; 37(24):5471-8.

30. Kjaer J, Olsen P, Bach K, Barlebo HC, Ingerslev F, Hansen M et al. Leaching of estrogenic hormones from manure-treated structured soils. Environ. Sci. Technol. 2007; 41(11):3911-17.

31. Matthiessen P, Arnold D, Johnson AC, Pepper TJ, Pottinger TG, Pulman KGT. Contamination of headwater streams in the United Kingdom by estrogenic hormones from livestock farms. Sci. Total Environ. 2006; 367(2-3):616-30.

32. Carballa M, Omil F, Lema JM, Llompart M, Garcia-Jares C, Rodríguez I, et al. Behavior of pharmaceuticals, cosmetics and hormones in a sewage treatment plant. Water Res.2004; 38:2918-26.

33. Stasinakis AS, Kordoutis CI, Tsiouma VC, Gatidou G, Thomaidis NS. Removal of selected endocrine disrupters in activated sludge systems: Effect of sludge retention time on their sorption and biodegradation. Bioresour. Technol. 2010; 101(7):2090-5.

34. Chen CY, Wen TY, Wang GS, Lin YH, Lien GW. Determining estrogenic steroids in Taipei waters and removal in drinking water treatment using high-flow solid-phase extraction and liquid chromatography/tandem mass spectrometry. Sci. Total Environ. 2007; 378(3):352-65.

35. Gross M, Petrovic M, Barceló D. Waste water treatment plants as a pathway for aquatic contamination by pharmaceuticals in the Ebro river basin (Northeast Spain). Environ. Toxicol. Chem. 2007; 26(8):1553-62.

36. Köck-Schulmeyer M, Ginebreda A, Postigo C, López-Serna R, Pérez S, Brix R, et al. Wastewater reuse in Mediterranean semi-arid areas: The impact of discharges of tertiary treated sewage on the load of polar micro pollutants in the Llobregat river (NE Spain). Chemosphere. 2011; 82:670-8.

37. Fine DD, Breidenbach GP, Price TL, Hutchins SR. Quantitation of estrogens in ground water and swine lagoon samples using solid-phase extraction, pentafluorobenzyl / trimethylsilyl derivatizations and gas chromatography negative ion chemical ionization tandem mass spectrometry. J. Chromatogr., A. 2003; 1-2(1017):167-85.

38. Furuichi T, Kannan K, Suzuki K, Tanaka S, Giesy JP, Masunaga S. Occurrence of estrogenic compounds in and removal by a swine farm waste treatment plant. Environ. Sci. Technol. 2006; 40:7896902.

39. Sarmah AK, Meyer MT, Boxall ABA. A global perspective on the use, sales, exposure pathways, fate and effects of veterinary antibiotics (VAs) in the environment. Chemosphere. 2006; 65:725-59.

40. Raman DR, Williams EL, Layton AC, Burns RT, Easter JP, Daugherty AS, et al. Estrogen content of dairy and swine wastes. Environ. Sci. Technol. 2004; 13(38):3567-73. 
41. Kunz A, Oliveira PA, Higarashi MM, Sangoi V. Recomendações técnicas para uso de esterqueiras para a armazenagem de dejetos de suínos. Comunicado Técnico. 2004; 361(1-4).

42. Bradford SA, Segal E, Zheng W, Wang QQ, Hutchins SR. Reuse of concentrated animal feeding operation wastewater on agricultural lands. J. Environ. Qual. 2008; 37(3):S97-S115.

43. Ying GG, Toze S, Hanna J, Yu XY, Dillon P, Kookan RS. Decay of endocrine-disrupting chemicals in aerobic and anoxic groundwater. Water Res., 2008; 42(4-5):1133-41.

44. Ying GG, Kookana RS, Kumar A, Mortimer M. Occurrence and implications of estrogens and xenoestrogens in sewage effluents and receiving waters from South East Queensland. Sci. Total Environ. 2009; 407(18):5147-55.

45. Pinheiro A, Rosa Albano RM, Alves TC, Kaufmann V, Da Silva MR. Veterinary antibiotics and hormones in water from application of pig slurry to soil. Agric. Water Manage. 2013; 129:1-8.

46. Vega-MOrales T, Sosa-Ferrera Z, Santana-Rodríguez JJ. Determination of alkylphenol polyethoxylates, bisphenol-A, 17a-ethynylestradiol and $17 \beta$-estradiol and its metabolites in sewage samples by SPE and LC/MS/MS. J. Hazard. Mater. 2010; 183(1-3): 701-11.

47. Jelic A, Meritxell G, Ginebreda A, Cesperes-Sanchez R, Ventura F, Petrovic M, et al. Occurrence, partition and removal of pharmaceuticals in sewage water and sludge during wastewater treatment. Water Res. 2011; 45(3):1165-76.

48. Almeida C, Nogueira JMF. Determination of steroid sex hormones in water and urine matrices by stir bar sorptive extraction and liquid chromatography with diode array detection. J. Pharm. Biomed. Anal. 2006; 41(4):1303-11.

49. Ribani M, Bottoli CBG, Collins CH, Jardim ICSF, Melo LFC. Validação em métodos cromatográficos e eletroforéticos. Quím. Nova. 2004; 27:771-80.

50. Bastos RKX, Bevilacqua PD, Mierzwa JC. Análise de risco aplicada ao abastecimento de água para consumo humano. In: Pádua VL, coordenador. Remoção de microrganismos emergentes e micro contaminantes orgânicos no tratamento de água. Rio de Janeiro: ABES; 2009. p. 327-60.

51. Reis Filho RW, Araújo JC, Vieira EM. Hormônios sexuais estrógenos: Contaminantes bioativos. Quím. Nova. 2006; 29(4):817-22.

52. Hutchins SR, White MV, Hudson FM, Fine DD. Analysis of lagoon samples from different concentrated animal feeding operations for estrogens and estrogen conjugates. Environ. Sci. Technol. 2007; 3(41):738-44.

53. Ferreira MGM. Remoção da atividade estrogênica de $17 \beta$-estradiol e de $17 \alpha$-etinilestradiol pelos processos de ozonização [tese]. Rio de Janeiro: Universidade Federal do Rio de Janeiro; 2008. 
54. Baronti C, Curini R, Ascenzo GD, Corcia AD, Gentili A, Chimica D. Monitoring natural and synthetic estrogens at activated sludge sewage treatment plants and in a receiving river water. Environ. Sci. Technol. 2000; 24(34):5059-66.

55. Clara M, Kreuzinger N, Strenn B, Gans O, Kroiss H. The solids retention time: a suitable design parameter to evaluate the capacity of wastewater treatment plants to remove micropollutants. Water Res. 2005; 39:97-106.

56. Johnson AC, Aerni HR, Gerritsen A, Gibert M, Giger W, Hylland K, et al. Comparing steroid estrogen, and nonylphenol content across a range of European sewage plants with different treatment and management practices. Water Res. 2005; 39:47-58.

57. Joss A, Andersen H, Ternes T, Richle PR, Siegrist H. Removal of estrogens in municipal wastewater treatment under aerobic and anaerobic conditions: consequences for plant optimization. Environ. Sci. Technol. 2004; 38:3047-55.

58. Silva CGA, Collins CH. Aplicações de cromatografia líquida de alta eficiência para o estudo de poluentes orgânicos emergentes. Quím. Nova. 2011; 34(4):665-76.

59. Smith CA. Normal and abnormal parturition in swine. In: Youngquist RS, editor. Current therapy in large animal theriogenology. Philadelphia: W. B. Saunders Company; 1997. p. 719-26.

60. First NL, Lohse JK, Nara BS. The endocrine control of parturition. In: Cole DJA, Foxcroft GR, editors. Control of pig reproduction. London: Butterworth Scientific; 1982. p. 311-42.

61. Anderson LL. Pigs. In: Hafez ESE, editor. Reproduction in farm animals. 6th ed. Philadelphia: Lea \& Febiger; 1993. p. 343-60.

62. Lu GH, Song WT, Wang C, Yan ZH. Assessment of in vivo estrogenic response and the identification of environmental estrogens in the Yangtze River (Nanjing section). Chemosphere. 2010; 9(80):982-90.

63. Zuo Y, Zhang K, Deng Y. Occurrence and photochemical degradation of $17 a$-ethinylestradiol in acushnet river estuary. Chemosphere. 2006; 63:1583-90.

64. Gadd JB, Tremblay LA, Northcott GL. Steroid estrogens, conjugated estrogens and estrogenic activity in farm dairy shed effluents. Environ. Pollut. 2010; 158(3): 730-6.

\section{Agradecimentos}

Gostaríamos de agradecer a FAPESC, Termos de Outorga 17419/2011-0 e TR2012000488, ao CNPq, Processo 562378/2010-3, Edital 22/2010 - Tem C, pelo suporte financeiro ao desenvolvimento do trabalho, ao CNPq, processo 302022/2011-2, pela bolsa de produtividade de pesquisa, à CAPES pela concessão da bolsa de mestrado e aos proprietários das Granjas, Edivaldo da Costa, Olmir Paulinho Benjamini e Ademir Sachine, que permitiram a realização deste trabalho. 